\title{
The Role of N-Acetylcysteine in Inhibiting Responding During Extinction in Rats Trained to Self-Administer Cocaine
}

\author{
Steven D. LaRowe ${ }^{*}, 1,2$ and Peter W. Kalivas ${ }^{3}$ \\ ${ }^{1}$ Substance Abuse Treatment Center, Mental Health Service Line, Ralph H. Johnson, VAMC, Charleston, SC. 29401, \\ USA \\ ${ }^{2}$ Department of Psychiatry and Behavioral Sciences, Medical University of South Carolina, Charleston, SC 29425, USA \\ ${ }^{3}$ Department of Neurosciences, Medical University of South Carolina, Charleston, SC 29425, USA
}

\begin{abstract}
Absract: Previous work has found that $\mathrm{N}$-acetylcysteine inhibits extinction responding in rats trained to self-administer heroin. The current study examined the ability of $\mathrm{N}$-acetylcysteine to inhibit extinction responding in rats trained to selfadminister cocaine. Rats were trained to self-administer cocaine $(0.39 \mathrm{mg} / \mathrm{kg})$ for 10 to 12 days and were pretreated with either $\mathrm{N}$-acetylcysteine $(60 \mathrm{mg} / \mathrm{kg})$ or saline beginning on the first day of extinction training and on each extinction training day thereafter. Results indicated that chronically administered $\mathrm{N}$-acetylcysteine reduced lever pressing during extinction sessions. In addition to demonstrating the impact $\mathrm{N}$-acetylcysteine has on lever pressing during extinction, the present study underscores the importance of using responding during extinction as a dependent measure in the development of medications for addictive behaviors.
\end{abstract}

Keywords: Cocaine, addiction, $\mathrm{N}$-acetylcysteine, glutamate, extinction, cystine-glutamate exchanger.

\section{INTRODUCTION}

Despite over 15 years of on-going research efforts, an FDA-approved medication for the treatment of cocaine dependence remains elusive. Nevertheless, the mechanisms underlying cocaine dependence and treatments based on those mechanisms are now being explored using animal models of addiction $[1,2]$. One widely used model is the extinction-reinstatement model of drug-seeking, which putatively models the process of relapse in human addicts [3]. In this model, animals learn to self-administer drugs of abuse. This behavior is extinguished, and then reinstated with either a priming dose of drug, drug-related cues or a stressor. In addition to providing a means to study the basic mechanisms underlying cocaine addiction, this model provides a means to screen novel pharmacological compounds to test for their ability to block reinstatement.

$\mathrm{N}$-acetylcysteine (NAC) is one compound that has shown efficacy in reducing cocaine-induced reinstatement $[4,5]$. Nacetylcysteine stimulates cystine-glutamate exchange and restores basal levels of glutamate within the nucleus accumbens [4]. This normalization of glutamatergic tone presumably allows for normal activation of glutamatergic autoreceptors within the accumbens, thereby regulating what would be otherwise excessive release of glutamate during cocaine-induced reinstatement.

Within the cocaine literature, a limitation of previous work within the extinction-reinstatement paradigm is that administration of reinstatement-blocking agents is frequently

\footnotetext{
*Address correspondence to this author at the Department of Psychiatry and Behavioral Sciences, Medical University of South Carolina, 67 President St., MSC 861, Medical University of South Carolina, Charleston, SC 29425, USA; Tel: 843-789-6316; Fax: 843-792-6316; E-mail: larowe@musc.edu
}

acute, usually occurring hours or even minutes before drug or cue challenge [4-7]. In clinical settings, however, medications are usually not administered on an acute basis, but rather as part of a chronic (i.e., daily) treatment regimen $[8,9]$. It is feasible that a drug that is found to be effective after acute administration may not be as effective if delivered chronically. To more closely model what occurs in clinical settings, it is necessary to examine the effects of medications in the context of both acute and chronic regimens.

In an attempt to model the chronic nature of clinical treatment regimens, Zhou and Kalivas [10] used the extinction-reinstatement paradigm to examine the impact of chronically administered NAC in rats trained to administer heroin. After self-administration training, rats received a chronic treatment regimen consisting of daily treatments of NAC or saline starting on the first day of extinction training and continuing throughout. Results revealed that NAC, delivered on a chronic basis, not only reduced reinstatement of heroin-seeking behavior, but also inhibited responding (i.e., lever pressing) during extinction sessions. Thus, the introduction of a chronic treatment regimen into the extinction-reinstatement paradigm revealed that responding during extinction, in addition to reinstatement, may serve as a potentially important dependent variable worthy of investigation in its own right. Given these findings, it is reasonable to hypothesize that chronically administered NAC will reduce responding during extinction in rats trained to self-administer cocaine. The present report consists of pilot study designed to investigate this hypothesis.

\section{MATERIALS AND METHODOLOGY}

\section{Subjects}

Male Sprague-Dawley rats (Charles River, Wilmington, MA) were individually housed in a temperature- and 
humidity-controlled colony room on a 12 hour light/dark cycle. Rats were maintained on 25-30g rat chow (Harlan, Indianapolis, IN) per day and ad libitum water. The housing and care of the rats followed the guidelines of the Guide for the Care and Use of Laboratory Rats (Institute of Laboratory Animal Resources on Life Sciences, National Research Council, 1996). All procedures were approved by the Medical University of South Carolina's Institutional Animal Care and Use Committee.

\section{Surgery}

Rats were anesthetized using a mixture of ketamine HCL (Fort Dodge Animal Health, Fort Dodge, IA) and xylazine (Phoenix Pharmaceutical, St. Joseph, MO); $66 \mathrm{mg} / \mathrm{kg}$ and $1.33 \mathrm{mg} / \mathrm{kg}$, respectively, followed by equithesin (Sigma, St. Louis, MO, $0.5 \mathrm{ml} / \mathrm{kg}$ of a solution of $9.72 \mathrm{mg} / \mathrm{ml}$ pentobarbital sodium $42.5 \mathrm{mg} / \mathrm{ml}$ chloral hydrate and 21.3 $\mathrm{mg} / \mathrm{ml}$ magnesium sulfate heptahydrate dissolved in a $44 \%$ propylene glycol, $10 \%$ ethanol solution; IP). Chronic indwelling catheters were constructed using a bent steel cannula with a screw-type connector (Plastics One, Roanoke, VA), SILASTIC tubing $(10 \mathrm{~cm}$, i.d. $0.64 \mathrm{~mm}$, o.d. $1.19 \mathrm{~mm}$; Dow Corning, Midland, MI), Prolite polypropylene monofilament mesh (Atrium Medical Corporation, Hudson, $\mathrm{NH}$ ), and cranioplastic cement (Lang Dental Manufacturing, Wheeling, IL), as described previously [11]. The end of the catheter was inserted into the right jugular vein and was secured to surrounding tissue with suture. The catheter ran subcutaneously and exited on the rat's back, posterior to the shoulder blades.

To extend catheter patency, the catheters were flushed once daily for three days following surgery with $0.1 \mathrm{ml}$ of an antibiotic solution of cefazolin $(10.0 \mathrm{mg} / \mathrm{ml}$, Schein Pharmaceutical, Florham Park, NJ) dissolved in heparinized saline $(70 \mathrm{U} / \mathrm{ml}$; Elkins-Sinn, Cherry Hill, NJ). Thereafter, catheters were flushed prior to each self-administration session with $0.091 \mathrm{ml}$ of heparinized saline $(10 \mathrm{U} / \mathrm{ml})$, and subsequently flushed with $0.1 \mathrm{ml}$ of the above-described cefazolin solution and $0.1 \mathrm{ml}$ of heparinized saline at the end of each session. Catheter patency was periodically verified by infusing $0.12 \mathrm{ml}$ of methohexital sodium $(10 \mathrm{mg} / \mathrm{ml}$, IV; Eli Lilly, Indianapolis, IN), which produces a rapid loss of muscle tone only when administered intravenously.

\section{Self-Administration and Extinction Training}

Rats were allowed a minimum of five days to recover from surgery. Rats were trained to lever press on a fixed ratio one (FR 1) schedule of food reinforcement (45 mg pellets; Research Diets, New Brunswick, NJ) in soundattenuated, operant conditioning chambers $(30 \times 20 \times 24 \mathrm{~cm}$ high; Med Associates, St. Albans, VT) during a 15 hour overnight food training session. The chambers were equipped with two retractable levers, a stimulus light above the active lever, a food pellet dispenser, and a house light. Experimental events and data collection were controlled by Schedule Manager software version 3.13 (Med Associates). During the session, each lever press on the right resulted in delivery of a food pellet only (i.e., active lever). Lever presses on the left lever had no programmed consequences (i.e., inactive lever). Rats were required to earn 100 pellets or more during the session and exhibit $50 \%$ more responding on the active lever than the inactive lever.
Self-administration training was conducted during twohour daily sessions during the rats' dark cycle. Rats were trained to press a lever according to a FR 1 schedule of cocaine reinforcement (cocaine hydrochloride; National Institute on Drug Abuse, Research Triangle Park, NC). Each infusion was followed by a 20 -second time-out period, during which time the active lever had no programmed consequences. Six rats were trained to self-administer 0.13 $\mathrm{mg}$ per each $0.10 \mathrm{ml}$ infusion $(\sim 0.39 \mathrm{mg} / \mathrm{kg})$. Each infusion was accompanied by a five second presentation of a stimulus light above the active lever. Subjects were required to selfadminister at least 10 infusions on 10 occasions in order to meet self-administration criteria, and all subjects achieved this criterion between 10 and 14 sessions. Throughout all training sessions, a house light was illuminated. Following self-administration training, rats underwent two-hour daily extinction sessions, during which responses were recorded on both levers, but had no programmed consequences. On day one of extinction, each rat was assigned to a chronic treatment condition, consisting of either saline or an injection of NAC $(60 \mathrm{mg} / \mathrm{kg})$, dissolved in saline and buffered with $\mathrm{NaOH}$ to achieve a $\mathrm{pH}$ level greater than 5.4. Daily treatment continued throughout the remainder of the experiment. Each subject completed seven extinction sessions.

\section{Analyses}

Daily extinction data were analyzed using a repeated measures ANOVA with Extinction Day as the repeated within-subjects variable and Treatment Condition (NAC vs Saline) as a between-subjects variable.

\section{RESULTS}

Data for self administration infusion levels did not differ with respect to treatment group, as the mean number of infusions for the NAC group was 45.02, $(S E=6.41)$, and $34.13(S E=8.67)$ for the Saline group, $p=0.37$. The repeated measures ANOVA revealed a main effect for Extinction Day, $F(6,24)=3.47, p<0.05$. The Extinction Day $\mathrm{x}$ Treatment Condition interaction showed a trend towards significance, $p<0.09$; moreover, there was a main effect for Treatment Condition, $F(1,4)=11.24, p<0.05$. The mean number of lever presses across extinction days was $8.95(S E=5.23)$ for the NAC group, which was significantly lower than that of the Saline group, which was 32.76 ( $S E=$ 5.23). The main effect for Treatment Condition and the individual daily levels of extinction responding (by treatment condition) are presented in Fig. (1).

In order to determine whether NAC was facilitating the rate at which extinction occurred (as opposed to merely reducing level of responding in general), the time course of responding was examined within the first session. Data within the first two-hour extinction session were divided into 15-minute blocks. The cumulative percentage of extinction responding was calculated at each 15 -minute time point. It was hypothesized that if NAC were facilitating the rate of extinction learning, there would be a leftward shift in the cumulative probability curve within the NAC group. A repeated measures ANOVA was performed, with Block as a within-subject repeated measure, and Treatment Condition as a between-subjects variable. While the expected effect for Block was significant, $F(7,28)=11.60, p<0.01$, the main 


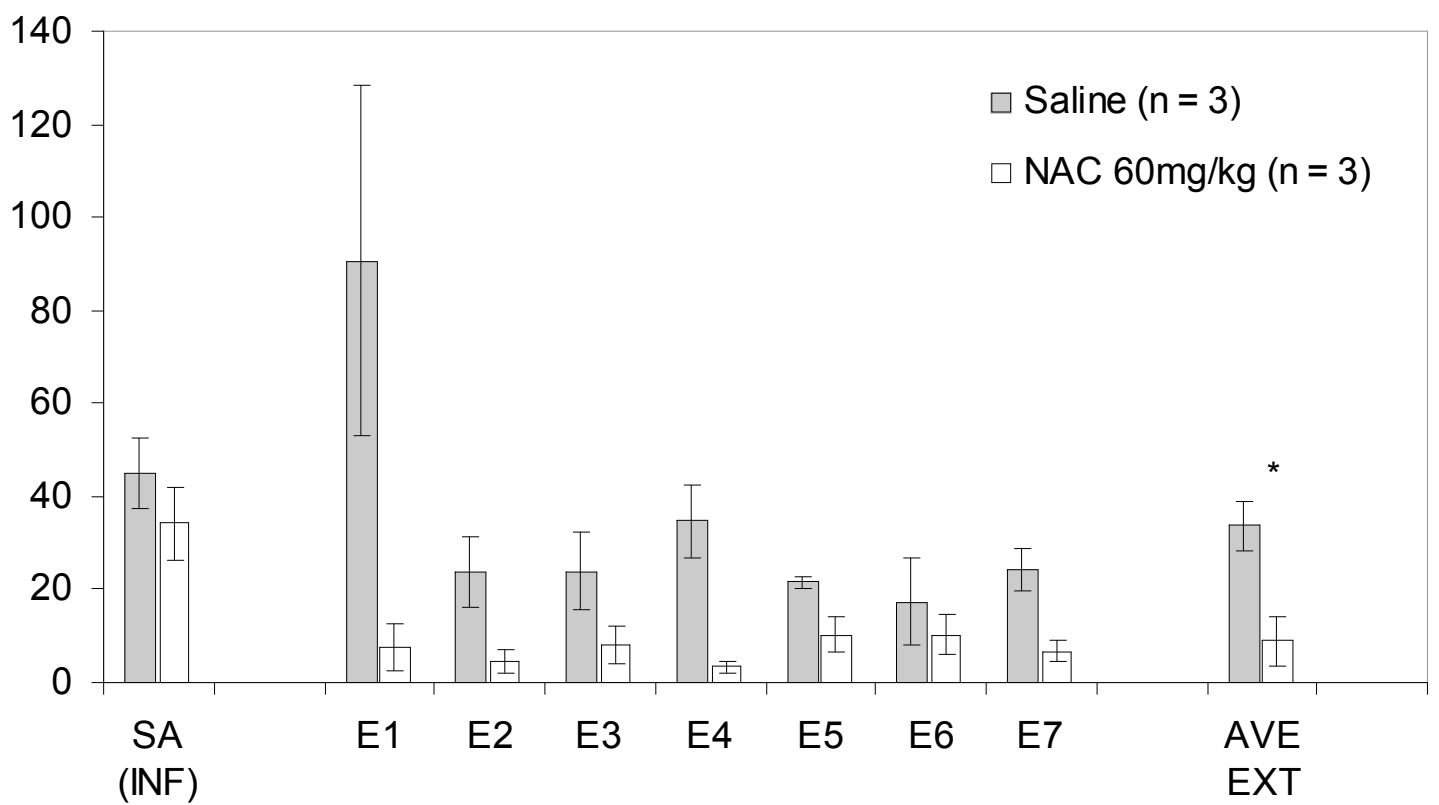

Fig. (1). Effect of NAC on extinction responding in animals trained to self-administer cocaine. Data are shown as mean \pm SEM per two hour training session. Self-Administration (SA INF) is represented as mean infusions; for daily extinction trials (E), data are shown as mean \pm SEM active lever presses. ${ }^{*} \mathrm{p}<0.05$; significance level pertains to the comparison of the overall main effect between NAC and Saline across all seven days of extinction training (AVE EXT).

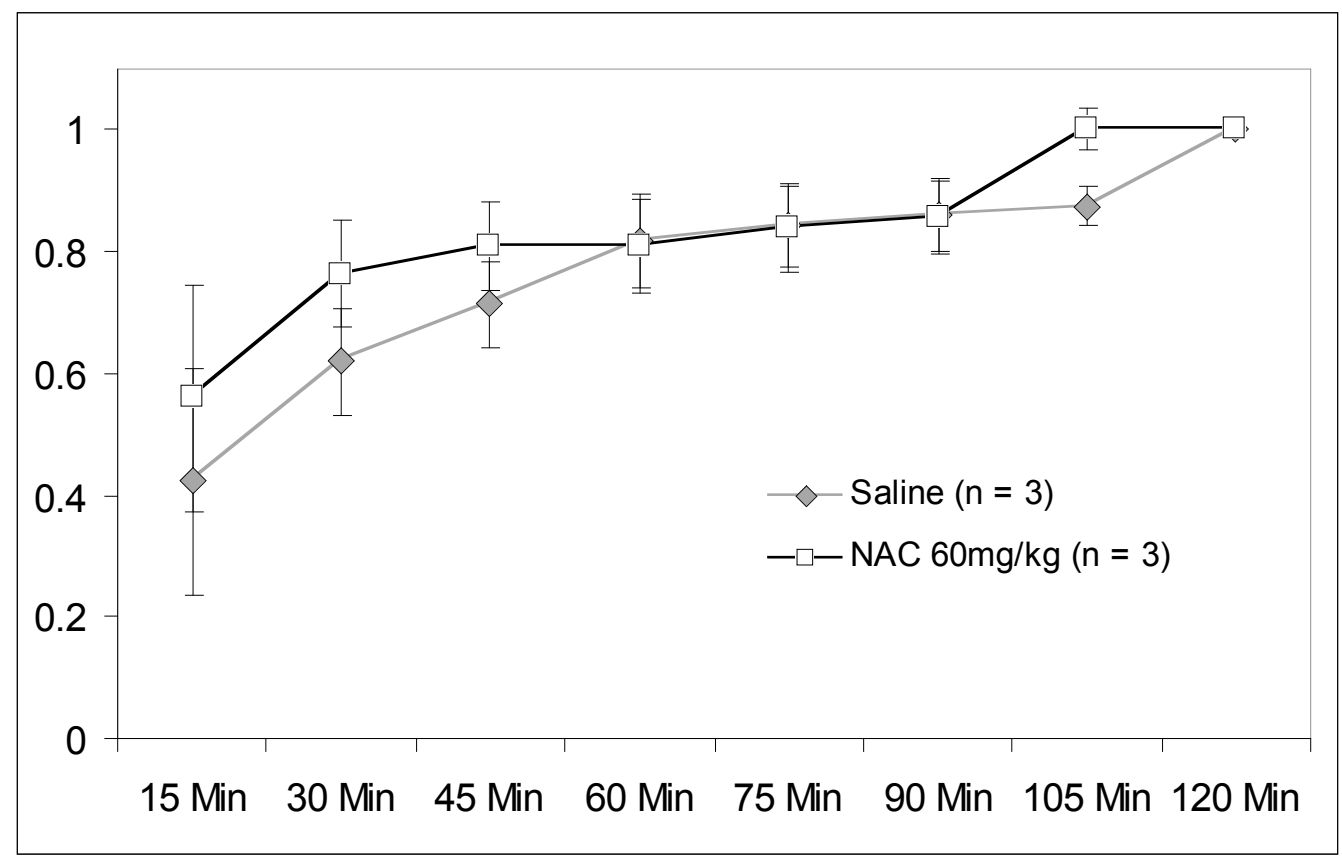

Fig. (2). Cumulative percent of extinction responding for the first two-hour extinction session. Data are shown as mean \pm SEM per 15 minute block within the two hour training session.

effect for Treatment Condition was not significant $(p=$ 0.54), nor was the Block $x$ Treatment Condition Interaction $(p=0.76)$. Cumulative probability data are presented in Fig. (2).

\section{DISCUSSION}

Akin to the previous study with heroin-trained animals [10], the findings of this study suggest that pretreatment with NAC was associated with reduced levels of lever presses during extinction sessions. It remains to be seen whether cocaine-seeking rats, like the heroin-seeking rats, display long-lasting effects on reinstatement as well. Although the mechanisms whereby NAC may reduce responding during extinction are not identified in this report, closer examination of the data did not provide evidence that NAC increased the rate of extinction during the first session of extinction training, arguing against the possibility that NAC enhances the rate of extinction learning per se. This suggests that NAC reduced responding levels overall, raising the possibility that NAC may be suppressing behavior or motivation in general. 
However, previous studies have shown that this dose of NAC does not affect reinstatement for food responding, nor does it reduce motivation to self-administer cocaine (4). Thus, the reduction in responding does not appear to be a result of general reduction in motivation or a result of an inability to engage in lever pressing.

It is known that returning an animal to the original context in which it learned to self-administer drugs can reinstate previously extinguished drug-seeking behavior [12]. Within the present extinction-reinstatement paradigm, the operant chamber itself serves as a contextual cue that can potentially reinstate cocaine-seeking behavior each time the animal is returned to it. Previous work has suggested that the dorsal hippocampus (DH), the basal lateral amygdala (BLA), and the dorsal medial prefrontal cortex (dmPFC) may be involved in this so-called contextual reinstatement of extinguished cocaine seeking behaviors [12]. These areas may also promote and maintain cocaine-seeking behaviors during extinction as well. It is known that glutamatergic pathways from these areas project into the core of the nucleus accumbens [1]. The accumbens core, which is generally believed to be deficient in basal levels of glutamate after chronic cocaine administration [4], becomes overactive when stimulated by the above-mentioned projections. This in turn maintains cocaine-seeking behaviors. Within the present study, NAC's purported ability to restore basal levels of glutamate in the accumbens may have reduced the contextinduced activation of the accumbens. Thus, it may be that by restoring glutamate levels in the accumbens, NAC may have reduced the impact of the motivational salience of the previously paired cocaine context (i.e., the operant chamber), which may in turn have reduced the level of responding within the NAC-treated animals during the extinction sessions.

\section{CONCLUSIONS}

The present report suggests that chronic NAC administration inhibits lever pressing during extinction sessions in rats trained to self-administer cocaine. It also represents an advance in "bench to bedside" translation, since daily administration of medication during extinction more closely models the treatment approaches used in clinical trials with human patients $[8,9]$. Compounds that impact cocaine seeking behaviors during extinction training in animal models may be candidate medications for promoting inhibition of drug-seeking behaviors in human addicts.

\section{ABBREVIATIONS \\ NAC $=$ N-acetylcysteine}

\section{ACKNOWLEDGEMENTS}

This research was made possible by support of NIDA Grants DA018501 and DA015369, and with co-operation of the Center for Drug and Alcohol Programs, Neurobiology of Addictions Research Center, and Division of Clinical Neurosciences, Medical University of South Carolina. Special thanks go to Joe Schacht who provided helpful comments during the revision of the manuscript.

\section{REFERENCES}

[1] Kalivas PW, Volkow ND. The neural basis of addiction: pathology of motivation and choice. Am J Psychiatry 2005; 162: 1403-13.

[2] Feltenstein MW, See RE. The neurocircuitry of addiction: an overview. Br J Pharmacol 2008; 154: 1-14.

[3] Shaham Y, Shalev U, Lu L, de Witt H, Stewart J. The reinstatement model of drug relapse: history, methodology and major findings. Psychopharmacology (Berl) 2003; 168: 3-20.

[4] Baker DA, McFarland K, Lake RW, et al. Neuroadaptation in cystine-glutamate exchange underlie cocaine relapse. Nat Neurosci 2003; 6:743-9.

[5] Moran MM, McFarland K, Melendez RI, Kalivas PW, Seamans JK. Cystine/glutamate exchange regulates metabotropic glutamate receptor presynaptic inhibition of excitatory transmission and vulnerability to cocaine seeking. J Neurosci 2005; 25: 6389-93.

[6] Kumaresana V, Yuana M, Yeea J, et al. Metabotropic glutamate receptor 5 (mGluR5) antagonists attenuate cocaine priming- and cue-induced reinstatement of cocaine seeking. Behav Brain Res 2009; 202: 238-44.

[7] Goeders NE, Clampitt DM, Keller C, Sharma M, Guerin GF. Alprazolam and oxazepam block the cue-induced reinstatement of extinguished cocaine seeking in rats. Psychopharmacology (Berl) 2009; 201: 581-8.

[8] Malcolm RJ, LaRowe SD, Cochran K, et al. A controlled trial of amlodipine for cocaine dependence: a negative report. J Subst Abuse Treat 2005; 28: 197-204.

[9] Mardikian P, LaRowe SD, Hedden S, Kalivas P, Malcolm R. An open-label trial of $\mathrm{N}$-acetylcysteine for the treatment of cocaine dependence: a pilot study. Prog Neuropsychopharmacol Biol Psychiatry 2007; 31: 389-94.

[10] Zhou W, Kalivas PW. N-Acetylcysteine reduces extinction responding and induces enduring reductions in cue- and heroinInduced drug-seeking. Biol Psychiatry 2008; 63: 338-40.

[11] Fuchs RA, See RE. Basolateral amygdala inactivation abolishes conditioned stimulus- and heroin-induced reinstatement of extinguished heroin-seeking behavior in rats. Psychopharmacology (Berl) 2002; 160: 425-33.

[12] Fuchs RA, Evans KA, Ledford CC, Parker MC, Case JM, Mehta $\mathrm{RH}$, See RE. The role of the dorsomedial prefrontal cortex, basolateral amygdala, and dorsal hippocampus in contextual reinstatement of cocaine seeking in rats. Neuropsychopharmacology 2005; 30: 296-309. 